\title{
Staged Energy Cascades for the LUX FEL
}

\author{
G. Penn \\ LBNL Technical Report: LBNL-55973 \\ July 2004
}

\begin{abstract}
Disclaimer
This document was prepared as an account of work sponsored by the United States Government. While this document is believed to contain correct information, neither the United States Government nor any agency thereof, nor The Regents of the University of California, nor any of their employees, makes any warranty, express or implied, or assumes any legal responsibility for the accuracy, completeness, or usefulness of any information, apparatus, product, or process disclosed, or represents that its use would not infringe privately owned rights. Reference herein to any specific commercial product, process, or service by its trade name, trademark, manufacturer, or otherwise, does not necessarily constitute or imply its endorsement, recommendation, or favoring by the United States Government or any agency thereof, or The Regents of the University of California. The views and opinions of authors expressed herein do not necessarily state or reflect those of the United States Government or any agency thereof, or The Regents of the University of California.
\end{abstract}

Ernest Orlando Lawrence Berkeley National Laboratory is an equal opportunity employer.

Prepared for the Department of Energy under contract number DE-AC03-76SF00098. 


\title{
STAGED ENERGY CASCADES FOR THE LUX FEL
}

\author{
G. PENN \\ LAWRENCE BERKELEY NATIONAL LABORATORY, BERKELEY, CA 94720
}

\begin{abstract}
Designs and simulation studies for harmonic cascades, consisting of multiple stages of harmonic generation in free electron lasers (FELs), are presented as part of the LUX R\&D project to design ultrafast, high photon energy light sources for basic science. Beam energies of 1.1, 2.1, and 3.1 GeV, corresponding to each pass through a recirculating linac, have independent designs for the harmonic cascade. Simulations were performed using the GENESIS FEL code, to obtain predictions for the performance of these cascades over a wide range of photon energies in terms of the peak power and laser profile. The output laser beam consists of photon energies of up to $1 \mathrm{keV}$, with durations of the order of $200 \mathrm{fs}$ or shorter. The contribution of shot noise to the laser output is minimal, however fluctuations in the laser and electron beam properties can lead to variations in the FEL output. The sensitivity of the cascade to electron beam properties and misalignments is studied, taking advantage of the fact that GENESIS is a fully 3-dimensional code.
\end{abstract}

\section{INTRODUCTION}

LUX $[1,2]$ is a design concept for an ultrafast X-ray science facility, based on an electron beam accelerated to $\mathrm{GeV}$ energies in a recirculating linac. Included in the design are short duration (200 fs or shorter FWHM) light sources which convert a $200-250 \mathrm{~nm}$ seed laser of similar duration to a much shorter wavelength through a harmonic cascade [3]. A harmonic cascade involves multiple stages of harmonic generation [4] in the low gain free electron laser (FEL) regime. In the LUX design, a harmonic cascade is used to produce very high harmonics of optical or UV lasers that would be tunable over a wide range of photon energies by varying the initial laser frequency and choosing a different sequence of harmonic numbers, which multiply the photon energy. The design considered here produces photon energies of up to $1 \mathrm{keV}$.

Harmonic generation has been demonstrated even in the high-gain regime [5]. Each stage of harmonic generation uses an incident radiation field to modulate those electrons within a bunch with which it overlaps inside a modulating undulator, or "modulator". The energy modulation is then passed through a dispersive region, in this case simply a set of adjustable dipoles which result in zero net kick but produce a time delay proportional to the relative energy deviation. If the energy modulation is larger than the intrinsic energy spread in the electron beam, the time delay can be chosen so as to create short microbunches within the beam. These microbunches contain Fourier components at harmonics of the initial wavelength. The electron beam then enters a second undulator, the "radiator", which is tuned to one of these harmonics. The microbunches will then radiate coherently at this harmonic well above the background level of radiation from noise. This radiation serves as the seed for the next stage of harmonic generation. The following modulator can thus have the same design as the radiator from the stage before, although in principle slight differences in tuning may be applied. As the energy spread has been increased in the portion of the electron beam which interacted with the laser field in the modulator, it is desirable to introduce a delay line after each radiator which causes the beam to slip behind the radiation field. In the next stage of the harmonic cascade, the region of overlap between the electron beam and the radiation field in the modulator will be in front of all previously affected portions of the electron beam. This delay line will typically include a large dispersion and chromaticity as well, which has the favorable side effect of flattening out the microbunching in previously used portions of the beam. Thus, even if the same electron beam is passed through another harmonic cascade, the "spoiled" portions of the electron beam will not produce radiation significantly above background levels.

Most of the undulators in the cascade operate in the low-gain FEL regime. Because of this, the spontaneous radiation levels remain very low and do not become amplified through the multiple harmonics, and the laser

Date: July 27, 2004. 
synchronizes the entire cascade. The output radiation can still vary due to fluctuations in the seed laser, electron beam, timing, or alignment. The sensitivity of the cascade to changes in emittance, energy spread, and current of the electron beam is studied for the three energy levels. The effect of misalignments on FEL performance is also examined

\section{Multiple Energy Level Configuration}

The recirculating linac accelerates the electron beam through multiple arcs. Each arc can include its own harmonic cascade tuned for that energy level, or even multiple cascades. Each cascade spoils an interval of the electron beam corresponding to several stages of harmonic generation, plus gaps in between. For initial laser widths of approximately $200 \mathrm{fs}$ FWHM, and for an electron bunch having a flat top current profile lasting about $2 \mathrm{ps,} \mathrm{it} \mathrm{may} \mathrm{be} \mathrm{possible} \mathrm{to} \mathrm{use} \mathrm{the} \mathrm{same} \mathrm{electron} \mathrm{bunch} \mathrm{for} \mathrm{several} \mathrm{harmonic} \mathrm{cascades.} \mathrm{The}$ laser must be synchronized to intersect with the electron bunch, preferably towards the tail of the bunch, in the first modulator. Subsequent delay lines will shift the radiation further towards the head of the bunch, where a previously undisturbed section of the electron bunch can be given an energy modulation, and radiate at a higher harmonic.

The main demand on the recirculating linac and arcs is to avoid generating any head-tail correlations, especially in beam energy, as well as preserving the intrinsic energy spread and emittance. The harmonic cascade consists of a series of undulators separated by alternating dispersive regions and delay lines, which will here be considered to have the same design and combine both functions. Any delay introduced in the dispersive section after each modulator will not affect the FEL performance because the radiation ceases to interact with the beam after the modulator; all timing and phase information is carried by the electrons. Similarly, the relative time shifts related to chromaticity will have no effect on the electron distribution except in regions that have experienced an energy modulation. The energy dependence of time-of-flight is characterized by the parameter $R_{56}$ where $c \Delta t=R_{56}\left(\gamma-\gamma_{0}\right) / \gamma_{0}$; here, $c$ is the speed of light, $\gamma m_{e} c^{2}$ is the particle energy, $m_{e}$ is the mass of the electron, and $\Delta t$ is the delay relative to the reference energy $\gamma_{0} m_{e} c^{2}$. Typical values for $R_{56}$ in the dispersive sections are $\leq 60 \mu \mathrm{m}$, while delays of $200-400$ fs will exhibit strong dispersive effects resulting in $R_{56}$ of order $100 \mu \mathrm{m}$. The delay lines control the timing in the cascade so long as the initial laser seed is correctly synchronized with the electron bunch. In addition, after each harmonic cascade the electron beam must be displaced so that the radiation can be refocused and carried to a beamline endstation. The electron beam can then be passed through another harmonic cascade, or it can be sent through a return arc back towards the linac.

In the following simulations, the laser seed is assumed to have a peak power of $100 \mathrm{MW}$ and FWHM of $200 \mathrm{fs}$, and to be tunable over a range of wavelengths including the interval from $200-250 \mathrm{~nm}$. Nominal values for the electron beam are $2 \mu \mathrm{m}$ normalized emittance, a uniform energy spread of $+/-200 \mathrm{keV}$, and a flat-top current profile having a value of $500 \mathrm{~A}$ and with a duration of $2 \mathrm{ps}$. Additional constraints on the phase of the laser seed, jitter in the laser parameters, beam alignments, and jitter in the beam parameters will be considered as part of the sensitivity studies. We also consider the effect of sub-nominal parameters on the performance of the harmonic cascades.

We consider a configuration where the beam makes three passes through the recirculating linac, exiting with energies of 1.1, 2.1, and $3.1 \mathrm{GeV}$. Tuning the wavelength of the initial laser seed allows for a $25 \%$ variation in the output photon energy. Choosing different integer harmonics allows for a large continuous range of photon energies to be produced. Proper FEL performance requires that the undulator satisfy the resonance condition, $\lambda \simeq \lambda_{U}\left(1+a_{U}^{2}\right) / 2 \gamma^{2}$, where $\lambda$ is the radiation wavelength, $\lambda_{U}$ is the undulator period, and $a_{U}$ is the normalized undulator field strength, defined as $a_{U} \equiv e B_{\mathrm{RMS}} \lambda_{U} / 2 \pi m_{e} c$, where $B_{\mathrm{RMS}}$ is the RMS value of the undulator field on axis. Thus, the undulators must be adjusted each time the output radiation wavelength is changed. The strength of the dispersive section should also be adjusted for optimal performance. At each energy level, the magnetic field in the final radiator must be increased as the desired wavelength is increased, leading to a maximum wavelength as the undulator reaches its peak strength. At very short wavelengths, the performance is degraded by a combination of large jumps in harmonic number from stage to stage, and degraded coupling between the electron beam and the radiation

field. If superconducting helical undulators are used for the final radiator, or if more advanced materials are used in a planar undulator, stronger magnetic fields and a larger span of wavelengths may be achievable. 
For the $1.1 \mathrm{GeV}$ electron beam, two harmonic generation stages are combined to produce radiation in the range of $10-31.3 \mathrm{~nm}$, using harmonic numbers $20,16,15,12,10$, and 8 . In addition, the sixth harmonic produces radiation in the range $33.3-41.7 \mathrm{~nm}$; the slight gap in wavelength requires a single harmonic of 7 to be used instead of two harmonic stages, or a wider range of seed laser wavelengths. For the $2.1 \mathrm{GeV}$ electron beam, three harmonic generation stages are combined to produce radiation in the range of $2.7-10.4$ $\mathrm{nm}$, using harmonic numbers $80,64,54,45,36,30$, and 24 . For the $3.1 \mathrm{GeV}$ electron beam, four harmonic generation stages are combined to produce radiation in the range of $1-2.7 \mathrm{~nm}$, using harmonic numbers 192, 160, 128, 108, and 90. For harmonic number 90, wavelengths up to $2.8 \mathrm{~nm}$ are possible but start to make high demands on magnet technology. The three energy levels combine to produce a continuous range of photon energies from $40 \mathrm{eV}$ up to $1.2 \mathrm{keV}$.

\section{Cascade Design}

Two different functions are performed by the undulators. The modulators act on those electrons within a longitudinal slice of the electron bunch that pass through the undulator simultaneously with an incident laser field. This yields an energy modulation and potentially generates bunching as well, although most of the modulators are sufficiently short so that the bunching effect is weak. In the low-gain regime, the initial laser seed determines the dynamics of the electrons and is in turn only weakly altered by the interaction, although the laser field is subject to diffraction as usual. Before the beam enters the next undulator, it is sent through a transport section that produces a correlation between longitudinal position and energy, denoted as $R_{56}$ where the time shift $\Delta t=\left(R_{56} / c\right)\left(\gamma-\gamma_{0}\right) / \gamma_{0}$, where $\gamma_{0} m_{e} c^{2}$ is the nominal energy. For appropriate values of $R_{56}$, this produces substantial bunching of the beam; if the energy modulation is sufficiently strong, this bunching will contain Fourier components at high harmonics of the initial modulation. However, the energy modulation is then transformed to an increase in energy spread, $\sigma_{\gamma}$, and in order for the following undulator to function properly the energy spread must satisfy $\sigma_{\gamma} / \gamma<\rho_{\mathrm{FEL}}$, where $\rho_{\mathrm{FEL}}$ is the "FEL parameter" [6] which reflects the tolerance of an FEL to deviations from the resonance condition. Additionally, the transverse emittance, $\epsilon_{N}$, should satisfy $\left(\epsilon_{N} / \gamma \lambda\right)<\left(4 \beta / \lambda_{U}\right) \rho_{\text {FEL }}, 1$, where $\beta$ is the beta function of the electron beam in the undulator. This second condition limits the photon energy which is practical for a given electron beam emittance and energy.

The beam then enters another undulator, the radiator, which is tuned to produce laser radiation at a higher harmonic of the initial laser seed. The electron beam radiates primarily because of the density modulation which has Fourier components at the appropriate wavelength; the radiation due to noise is extremely low, and the FEL instability is not important because the undulators operate in the low-gain regime where the total length of the undulator is smaller than the gain length. After exiting the radiator, the portion of the electron beam which has undergone the modulation and radiated now has a significantly enhanced energy spread and is no longer appropriate for further FEL interactions. Therefore, the electron beam is then delayed relative to the laser field using a chicane; in the following modulator, which is tuned to the same wavelength as the radiator preceding it, the laser field from the previous radiator overlaps a "fresh" section of the electron beam. The delay must be at least as large as the duration of the laser pulse, of order $200 \mathrm{fs}$.

The dispersive section, which produces the $R_{56}$ correlation, must be tuned to the appropriate strength in order to maximize the power produced by the radiator; it may also prove useful to adjust the delay line in order to choose the slices of the beam which will be interacting with the laser fields. In fact, both the dispersive section and delay line may be produced by a set of four dipoles; a single design can accomodate both needs. These sections have been designed by W. Wan and require a gap of $1.2 \mathrm{~m}$ between undulators. It is a good approximation to treat the electron beam transport through these sections as a combination of a drift region and a differential time delay.

The combination of a both an $R_{56}$ and a delay is not deleterious for either function. In fact, the additional, large $R_{56}$ within the delay line can damp out previous bunching generated in the electron beam and so reduce incidental radiation. These beam transport sections can fit within the $1.2 \mathrm{~m}$ drift between undulators; as length is at a premium, the beamline is made as simple as possible, at the cost of not being able to set independently $R_{56}$ and the delay. Typically, the $R_{56}$ parameter (with units of length) will be twice the increase in the nominal path length due to the chicane. The combination of the short distance with the fact that the undulators have similar natural focusing means that no additional matching is required; there are minor variations in beam size but they do not adversely affect the performance of the harmonic cascade. 


\section{UNDULATOR PARAMETERS}

Most of the undulators are assumed to be of the planar hybrid design, where magnetic pole pieces are sandwiched between alternating high-field permanent magnets. The peak magnetic field on axis is related to the gap, $g$, between undulators, according to the fit given by K. Halbach [7]:

$$
B_{0}[T]=a \exp \left[-\frac{g}{\lambda_{U}}\left(b-c \frac{g}{\lambda_{U}}\right)\right] .
$$

The parameters depend on the materials used in the magnet, and a separate set of calculations have been performed by P. Elleaume [8], using a 3D magnetostatic code. Different sets of parameters are given in Table 1; the first column, for $\mathrm{SmCo}_{5}$, with values $a=3.33, b=5.47, c=1.8$, is used here, and is conservative in that it generally predicts the smallest gap to obtain a given magnetic field. Tapering of the magnetic field is required to achieve proper matching for the electron beam, but this is not modelled in detail in the simulations. Instead, two additional magnetic periods on each side of the undulators are designated as dead space, with no magnetic field, as the fields near the endpoints should not contribute to the resonant interaction. The final radiators are taken to be helical undulators; they may be composed of superconducting coils, or permanent magnets. This allows for circularly polarized radiation and would also enhance the output power. Superconducting coils should be able to produce stronger magnetic fields (and so longer wavelengths) than the planar hybrid equivalents, but, for now, the field strength in the final undulators is assumed to be subject to the same constraints as that of the planar design.

TABLE 1. Parametric fit corresponding to Eq. 1 for hybrid undulator field strength as a function of gap and period, for different materials and models. The column corresponding to $\mathrm{SmCo}_{5}$ is used here to estimate the required gap.

\begin{tabular}{|c|cc|cc|}
\hline Model & \multicolumn{2}{|c|}{ K. Halbach } & \multicolumn{2}{c|}{ P. Elleaume } \\
Material & SmCo $_{5}$ & NdFeB & V permendur & NdFeB \\
\hline$a$ & 3.33 & 3.44 & 3.694 & 3.381 \\
$b$ & 5.47 & 5.08 & 5.068 & 4.730 \\
$c$ & 1.80 & 1.54 & 1.520 & 1.198 \\
\hline
\end{tabular}

The following undulator parameters are used: $140 \mathrm{~mm}$ period with minimum gap of $20 \mathrm{~mm}, 80 \mathrm{~mm}$ period with minimum gap $10 \mathrm{~mm}, 50 \mathrm{~mm}$ period with minimum gap $7.5 \mathrm{~mm}, 35 \mathrm{~mm}$ period with minimum gap 5 $\mathrm{mm}$, and $28 \mathrm{~mm}$ period with minimum gap $5 \mathrm{~mm}$. These undulators are common to all three energy levels, except the $140 \mathrm{~mm}$ period undulators are only used for 2.1 and $3.1 \mathrm{GeV}$ beams, and the $28 \mathrm{~mm}$ period undulator is only used as the final radiator for the $3.1 \mathrm{GeV}$ beam. For the $3.1 \mathrm{GeV}$ beam, the $50 \mathrm{~mm}$ period undulator may require a smaller gap in order to be useful for the 12th harmonic of the laser seed. However, this harmonic number can be avoided without serious repercussions, for example harmonic number 108 can be reached in the following progression: $6-18-54-108$, instead of using $4-12-36-108$, even though this requires a higher jump at the first stage. Even if the final radiator is designed to reach longer wavelengths, the second to last radiator can only produce wavelengths shorter than $6.8 \mathrm{~nm}$. Effectively, this means that the cascade will either be limited to wavelengths below $3.4 \mathrm{~nm}$, or the last two stages in the cascade will have to be designed for the same wavelength (i.e., harmonic number equal to unity), which is inefficient. So, in order to produce photon energies below $360 \mathrm{eV}$ with the $3.1 \mathrm{GeV}$ cascade, the $35 \mathrm{~mm}$ undulator will have to be replaced with a longer period undulator.

The undulator parameters for the harmonic cascades at 1.1, 2.1, and $3.1 \mathrm{GeV}$ are given in Tables 2 and 3. For planar hybrid undulators, the peak magnetic field on axis is tuned by adjusting the gap between the upper and lower sections of the magnetic array; helical undulators using superconducting coils will probably be tuned by varying the current. For the $3.1 \mathrm{GeV}$ harmonic cascade, it may be desirable to produce output radiation at wavelengths up to $3.13 \mathrm{~nm}$, instead of $2.67 \mathrm{~nm}$; this would require $a_{u}$ of up to 2.69 , and field on axis as high as $1.03 \mathrm{~T}$. Achieving this range in wavelength may require increasing the undulator period to $30 \mathrm{~mm}$, which typically reduces output power by $10 \%$.

Table 4 lists a set of harmonic sequences which cover the desired range of frequencies, given a seed laser which is tunable over the range $200-250 \mathrm{~nm}$. Note that there is a gap between 31.25 and $33.33 \mathrm{~nm}$, which 
TABLE 2. Planar undulator parameters and requirements for harmonic cascades at different energies. Lengths do not include tapering at endpoints.

\begin{tabular}{|c|c|c|c|c|c|}
\hline Period $(\mathrm{mm})$ & Beam Energy $(\mathrm{GeV})$ & length $(\mathrm{m})$ & $a_{u}$ & peak $B$ on axis $(\mathrm{T})$ & gap $(\mathrm{mm})$ \\
\hline 140 & 2.1 & 2.8 & $6.87-7.70$ & $0.74-0.83$ & $39-43$ \\
& 3.1 & 2.8 & $10.21-11.42$ & $1.10-1.24$ & $27-31$ \\
\hline 80 & 1.1 & 2.4 & $4.71-5.29$ & $0.89-1.00$ & $19-22$ \\
& 2.1 & 3.2 & $3.62-5.04$ & $0.68-0.95$ & $19-26$ \\
& 3.1 & 2.4 & $5.45-7.52$ & $1.03-1.42$ & $13-19$ \\
\hline 50 & 1.1 & 3.0 & $2.53-3.80$ & $0.77-1.15$ & $10.4-14.9$ \\
& 2.1 & 6.0 & $2.40-3.62$ & $0.73-1.10$ & $11.0-15.5$ \\
& 3.1 & 4.0 & $3.70-4.85$ & $1.12-1.47$ & $7.8-10.7$ \\
\hline 35 & 3.1 & 6.3 & $2.06-3.49$ & $0.89-1.51$ & $5.3-9.3$ \\
\hline
\end{tabular}

TABLE 3. Helical undulator parameters for the final radiating undulator in each cascade. Lengths do not include tapering at endpoints.

\begin{tabular}{|c|c|c|c|c|}
\hline Period $(\mathrm{mm})$ & Beam Energy $(\mathrm{GeV})$ & length $(\mathrm{m})$ & $a_{u}$ & $B$ on axis $(\mathrm{T})$ \\
\hline 35 & 1.1 & 6.3 & $1.28-3.17$ & $0.39-0.97$ \\
& 2.1 & 6.3 & $1.25-3.01$ & $0.38-0.92$ \\
\hline 28 & 3.1 & 8.4 & $1.32-2.45$ & $0.50-0.94$ \\
\hline
\end{tabular}

TABLE 4. Range of output wavelengths for different harmonic sequences, assuming a seed laser tunable between 200 and $250 \mathrm{~nm}$ wavelength.

\begin{tabular}{|c|l|c|}
\hline Energy $(\mathrm{GeV})$ & Harmonic Sequence & Wavelength Range $(\mathrm{nm})$ \\
\hline 1.1 & 36 & $33.33-41.67$ \\
& 48 & $25.00-31.25$ \\
& 510 & $20.00-25.00$ \\
& 412 & $16.67-20.83$ \\
& 515 & $13.33-16.67$ \\
& 416 & $12.50-15.63$ \\
& 520 & $8.00-12.50$ \\
\hline 2.1 & 41224 & $6.67-8.33$ \\
& 51530 & $5.56-6.94$ \\
& 41236 & $4.44-5.56$ \\
& 51545 & $3.70-4.63$ \\
& 61854 & $3.13-3.91$ \\
& 41664 & $2.50-3.13$ \\
\hline 3.1 & 52080 & $2.50-3.13$ \\
& 5204080 & $1.85-2.78$ \\
& 5154590 & $1.56-1.95$ \\
& 61854108 & $1.25-1.56$ \\
& 41664128 & $1.04-1.30$ \\
\hline & 52080160 & \\
\hline & 41664192 & \\
\hline
\end{tabular}

can be filled by using the 7th harmonic in a single-stage device, or by extending the laser wavelength, either down to $187 \mathrm{~nm}$ or up to $267 \mathrm{~nm}$. The rest of the range of wavelengths, from 1.04 to $41.67 \mathrm{~nm}$, can be made available by this scheme. 


\section{Simulation Results}

Here we present simulation results for select examples of harmonic cascades. These simulations were performed using the GENESIS [9] simulation code, which is fully 3-dimensional, allowing for the inclusion of effects such as beam offsets or misalignments. Earlier simulations have been performed by W.M. Fawley using the GINGER code [10].

The cascades have been optimized to be as short as possible and still produce significant output power at the highest desired photon energy. As a rough optimization, the power produced by the radiators is proportional to the square of their length; the energy modulation produced in the next undulator is proportional to the electric field times the length of the modulator. Thus, the energy modulation is proportional to the product of the lengths of the modulator and the upstream radiator. To maximize the energy modulation as a function of total length thus demands roughly that the two lengths be equal; to keep the design as simple as possible, each modulator has exactly the same configuration as the previous radiator. Each cascade begins with a single modulator that has an external laser focused on it to seed and synchronize the FEL interaction. The laser will be tunable over the range $200-250 \mathrm{~nm}$, although this entire range will not be necessary in order to have most of the different harmonics overlap with each other. Following this are pairs of identical undulators, except for the final radiator which produces the output of the cascade and which will probably be a superconducting helical undulator in order to produce circularly polarized radiation.

The results given below were obtained through multiple simulations in single-slice mode, each simulation corresponding to a different slice in the electron bunch. The simulation results are summarized in terms of the output power, variations in the output phase on axis, and the total energy per pulse. In addition, the transverse mode structure of the output radiation is described in terms of the $M^{2}$ parameter, which is the ratio of the emittance of the laser to the minimum emittance, $\lambda / 4 \pi$. This parameter can also be described as the ratio of the maximum Rayleigh length for the given (in this case, virtual) waist diameter to the observed Rayleigh length. Associated with this quantity are the apparent location of the laser waist, and the Rayleigh length itself. In terms of power flux, the corresponding RMS width of the laser at the waist is $\left(\lambda M^{2} Z_{R} / 4 \pi\right)^{1 / 2}$.

The peak brilliance, which quantifies the maximum phase space density of photons, is also given, along with the relative bandwidth of the output radiation defined as the FWHM of the power spectrum. For a pure Gaussian longitudinal profile in time, the bandwidth satisfies $\sigma_{t} \sigma_{\omega} \geq 1 / 2$; by analogy with the transverse $M^{2}$, we define a longitudinal factor $L^{2} \equiv 2 \sigma_{t} \sigma_{\omega}$. More generally, the $L^{2}$ parameter will include the effect of a power profile that is not Guassian, as well as the effect of phase variations. For example, a flat-top power profile with no phase variations has $L^{2}=2$. With this definition, the brilliance for a given energy per pulse is reduced by a factor of $L^{2} M^{4}$, where there is a factor of $M^{2}$ for each transverse plane. The peak brilliance is then

$$
1.2073 \times 10^{31} \frac{1}{L^{2} M^{4}} \frac{U}{1 \mu \mathrm{J}}\left(\frac{1 \mathrm{~nm}}{\lambda}\right)^{2} /(\mathrm{mm} \cdot \operatorname{mrad})^{2}(0.1 \% \text { bandwidth }) \mathrm{s}
$$

5.1. 1.1 GeV Cascade. For a $1.1 \mathrm{GeV}$ electron beam, the parameters are as follows: an $80 \mathrm{~mm}$ period undulator, with length $2.4 \mathrm{~m}$, then a pair of $50 \mathrm{~mm}$ period undulators with length $3.0 \mathrm{~m}$, then the final radiator with period $35 \mathrm{~mm}$ and length $6.3 \mathrm{~m}$. Including the $1.2 \mathrm{~m}$ drifts and the tapering, the total length of the cascade is $19.2 \mathrm{~m}$. In addition to this configuration, a short, single-stage harmonic generation section could be used to produce even lower energy photons. The output power is shown in Figure 1 for the case of $10 \mathrm{~nm}$ wavelength, with a temporal structure determined by the initial laser seed which has a wavelength of $200 \mathrm{~nm}$, peak power of $100 \mathrm{MW}$, and FWHM of $200 \mathrm{fs}$. Even with a Gaussian input laser, the final radiator produces a flat-top laser profile in time, with $213 \mathrm{MW}$ of peak power and FWHM of $180 \mathrm{fs}$, with 35 fs ramps. The total energy per pulse is $39 \mu \mathrm{J}$. For comparison, the total energy in the initial seed laser pulse is $21 \mu \mathrm{J}$. The transverse laser $M^{2}=1.07$, with the laser waist located $2.1 \mathrm{~m}$ in front of the beginning of the final undulator, and the Rayleigh length is $5.0 \mathrm{~m}$. The phase variation is negligible, so $L^{2}=2$ because of the flat-top profile, and the peak brilliance is $2 \times 10^{30} /(\mathrm{mm} \mathrm{mrad})^{2}(0.1 \% \mathrm{bw}) \mathrm{s}$. The FWHM relative bandwidth is $2 \times 10^{-4}$.

5.2. 2.1 GeV Cascade. For a $2.1 \mathrm{GeV}$ electron beam, the parameters are as follows: a $140 \mathrm{~mm}$ period undulator, with length $2.8 \mathrm{~m}$, then a pair of $80 \mathrm{~mm}$ period undulators with length $4.0 \mathrm{~m}$, then a pair of 

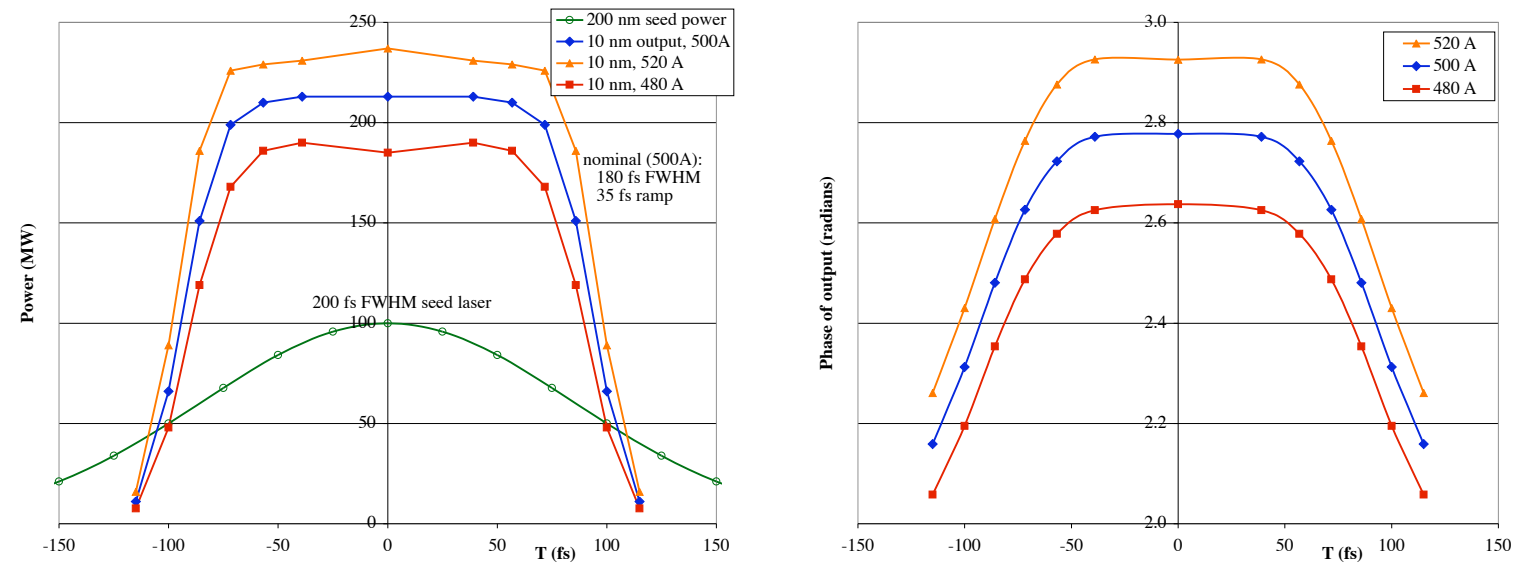

Figure 1. Temporal structure of output power and phase at $1.1 \mathrm{GeV}$, yielding $10 \mathrm{~nm}$ radiation from a $200 \mathrm{~nm}$ laser seed using harmonic numbers 5 and 20. Simulations assume an initial laser seed with $100 \mathrm{MW}$ peak power and with FWHM of $200 \mathrm{fs}$, for electron bunch current of $500 \mathrm{~A}$, and $500 \pm 20 \mathrm{~A}$.

$50 \mathrm{~mm}$ period undulators with length $5.0 \mathrm{~m}$, then the final radiator with period $35 \mathrm{~mm}$ and length $6.3 \mathrm{~m}$. Including the $1.2 \mathrm{~m}$ drifts and the tapering, the total length of the cascade is $34.8 \mathrm{~m}$. The output power is shown in Figure 2 for the case of $2.5 \mathrm{~nm}$ wavelength, with a temporal structure determined by the initial laser seed which has a wavelength of $200 \mathrm{~nm}$, peak power of $100 \mathrm{MW}$, and FWHM of $200 \mathrm{fs}$. Even with a Gaussian input laser, the final radiator produces a flat-top laser profile in time, with $35 \mathrm{MW}$ of peak power and FWHM of $145 \mathrm{fs}$, with $28 \mathrm{fs}$ ramps. The total energy per pulse is $5.0 \mu \mathrm{J}$. The transverse laser $M^{2}=1.27$, with the laser waist located $3.8 \mathrm{~m}$ in back of the beginning of the final undulator, and the Rayleigh length is $8.2 \mathrm{~m}$. The phase variation is approximately 1 radian, so $L^{2} \simeq 2.2$, and the peak brilliance is $3 \times 10^{30} /$ $(\mathrm{mm} \mathrm{mrad})^{2}(0.1 \% \mathrm{bw}) \mathrm{s}$. The FWHM relative bandwidth is $6 \times 10^{-5}$.
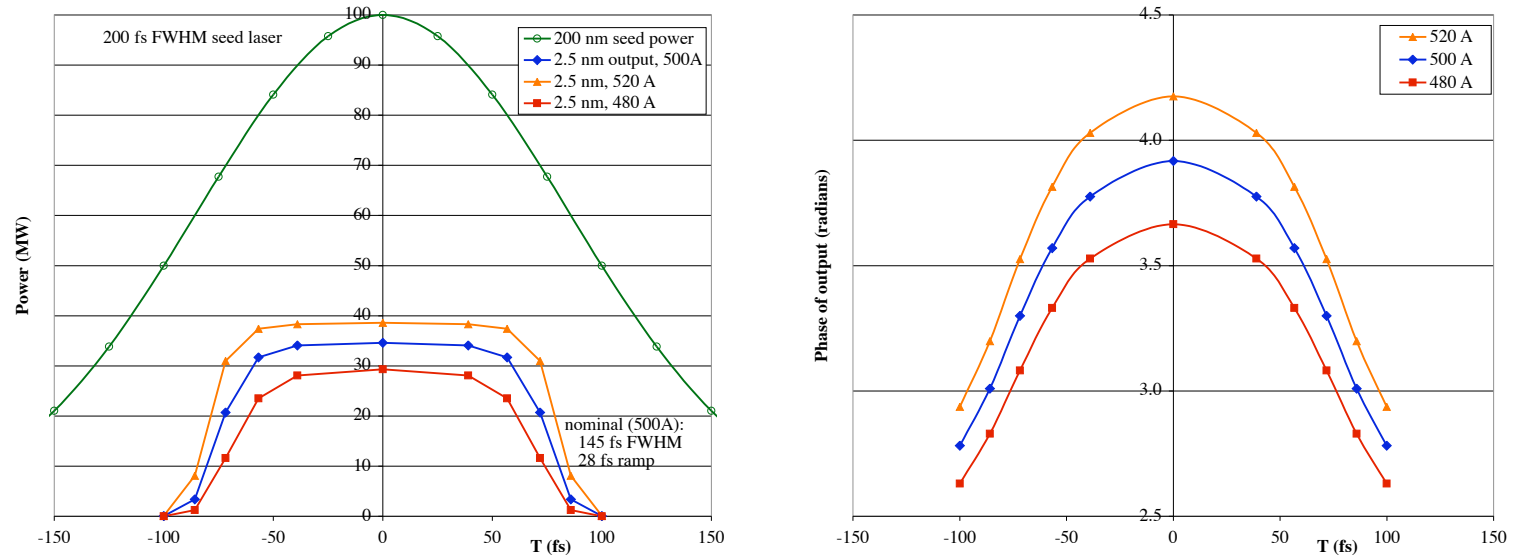

Figure 2. Temporal structure of output power and phase at $2.1 \mathrm{GeV}$, yielding $2.5 \mathrm{~nm}$ radiation from a $200 \mathrm{~nm}$ laser seed using harmonic numbers 5, 20, and 80. Simulations assume an initial laser seed with $100 \mathrm{MW}$ peak power and with FWHM of 200 fs, for electron bunch current of $500 \mathrm{~A}$, and $500 \pm 20 \mathrm{~A}$.

5.3. 3.1 GeV Cascade. For a $3.1 \mathrm{GeV}$ electron beam, the parameters are as follows: a $140 \mathrm{~mm}$ period undulator, with length $2.8 \mathrm{~m}$, then a pair of $80 \mathrm{~mm}$ period undulators with length $2.4 \mathrm{~m}$, then a pair of 
$50 \mathrm{~mm}$ period undulators with length $4.0 \mathrm{~m}$, then a pair of $35 \mathrm{~mm}$ period undulators with length $6.3 \mathrm{~m}$, then the final radiator with period $28 \mathrm{~mm}$ and length $8.4 \mathrm{~m}$. Including the $1.2 \mathrm{~m}$ drifts and the tapering, the total length of the cascade is $46.0 \mathrm{~m}$. The output power is shown in Figure 3 for the case of $1.0 \mathrm{~nm}$ wavelength, with a temporal structure determined by the initial laser seed which has a wavelength of 200 $\mathrm{nm}$, peak power of $100 \mathrm{MW}$, and FWHM of 200 fs. Even with a Gaussian input laser, the final radiator produces a flat-top laser profile in time, with $41 \mathrm{MW}$ of peak power and FWHM of $150 \mathrm{fs}$, with $16 \mathrm{fs}$ ramps. The total energy per pulse is $6.3 \mu \mathrm{J}$. The transverse laser $M^{2}=1.76$, with the laser waist located $5.5 \mathrm{~m}$ in back of the beginning of the final undulator, and the Rayleigh length is $12.2 \mathrm{~m}$. The phase variation is approximately 1.5 radians, so $L^{2} \simeq 2.7$, and the peak brilliance is $8 \times 10^{30} /(\mathrm{mm} \mathrm{mrad})^{2}(0.1 \% \mathrm{bw}) \mathrm{s}$. The FWHM relative bandwidth is $6 \times 10^{-5}$.

Another $3.1 \mathrm{GeV}$ example is presented, further from the emittance-limiting condition. The output radiation has been chosen at wavelength $2.0 \mathrm{~nm}$ using harmonic number 128 from a $250 \mathrm{~nm}$ laser seed. The output power is shown in Figure 4 with a temporal structure determined by the initial laser seed which has a wavelength of $250 \mathrm{~nm}$, peak power of $100 \mathrm{MW}$, and FWHM of $200 \mathrm{fs}$. Even with a Gaussian input laser, the final radiator produces a flat-top laser profile in time, with $353 \mathrm{MW}$ of peak power and FWHM of 160 fs, with 16 fs ramps. The total energy per pulse is $57 \mu \mathrm{J}$. The transverse laser $M^{2}=1.08$, with the laser waist located $2.5 \mathrm{~m}$ in back of the beginning of the final undulator, and the Rayleigh length is $11.2 \mathrm{~m}$. The phase variation is approximately 1 radian, so $L^{2} \simeq 2.2$, and the peak brilliance is $7 \times 10^{31} /(\mathrm{mm} \mathrm{mrad})^{2}$ $(0.1 \% \mathrm{bw}) \mathrm{s}$. The FWHM relative bandwidth is $4 \times 10^{-5}$.
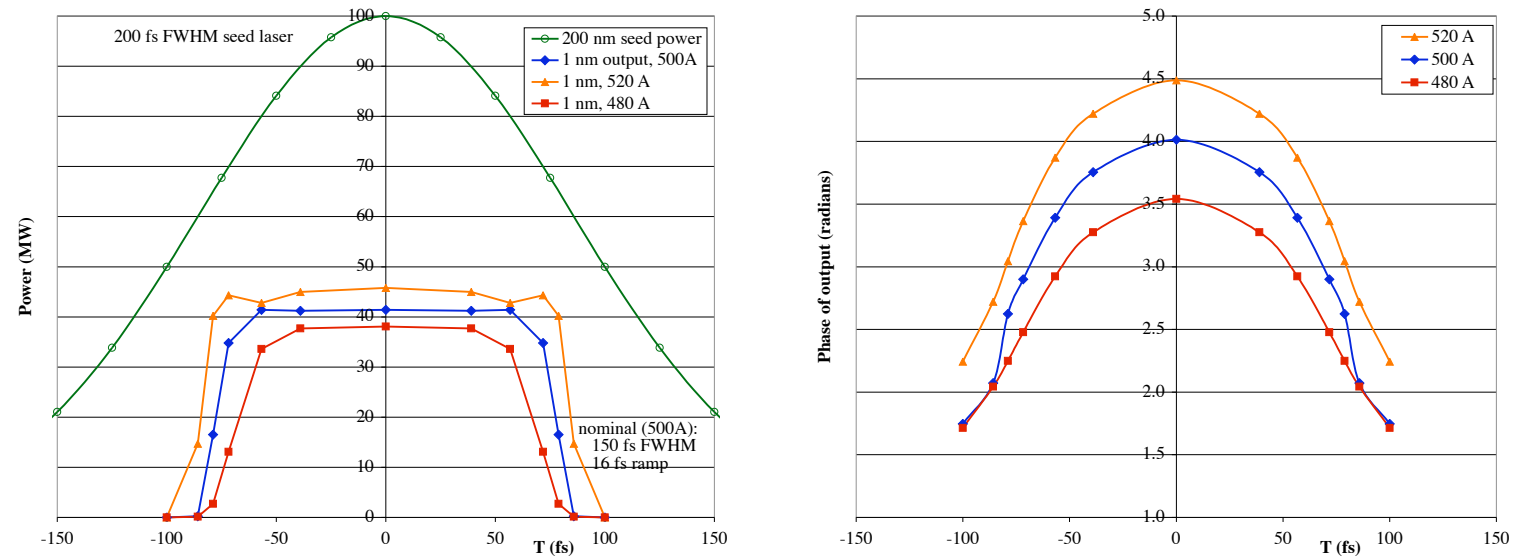

FiguRE 3. Temporal structure of output power and phase at $3.1 \mathrm{GeV}$, yielding $1.0 \mathrm{~nm}$ radiation from a $200 \mathrm{~nm}$ laser seed using harmonic numbers 4, 16, 64, and 192. Simulations assume an initial laser seed with $100 \mathrm{MW}$ peak power and with FWHM of $200 \mathrm{fs}$, for electron bunch current of $500 \mathrm{~A}$, and $500 \pm 20 \mathrm{~A}$.

5.4. Summary. The performance of each energy level at the highest photon energy has been described above. In Figure 5, the sweep of the cascades at each energy are shown, both as the laser frequency is adjusted and for different choices of harmonics. The output power rapidly improves as the wavelength is increased, reaching $700 \mathrm{MW}$ peak power for the $3.1 \mathrm{GeV}$ cascade, and well beyond $1 \mathrm{GW}$ peak power for the other cascades, until the wavelength becomes too long to be supported by the final undulator. Note that the bandwidths are determined by the temporal coherence of the initial laser seed and not by the number of undulator periods, so that relative bandwidths are expected to be of order $10^{-4}$. This enhances the peak brilliance, although experiments that are insensitive to variations in photon energy of this order will only be concerned with the total photon flux.

As an example of how the output frequency can be tuned over a wide range, consider the $2.1 \mathrm{GeV}$ beamline with an initial harmonic sequence $4-16$ - 64, with a seed laser having $200 \mathrm{~nm}$ wavelength. By increasing the wavelength of the seed laser up to $250 \mathrm{~nm}$, and adjusting the field strength in the undulators 

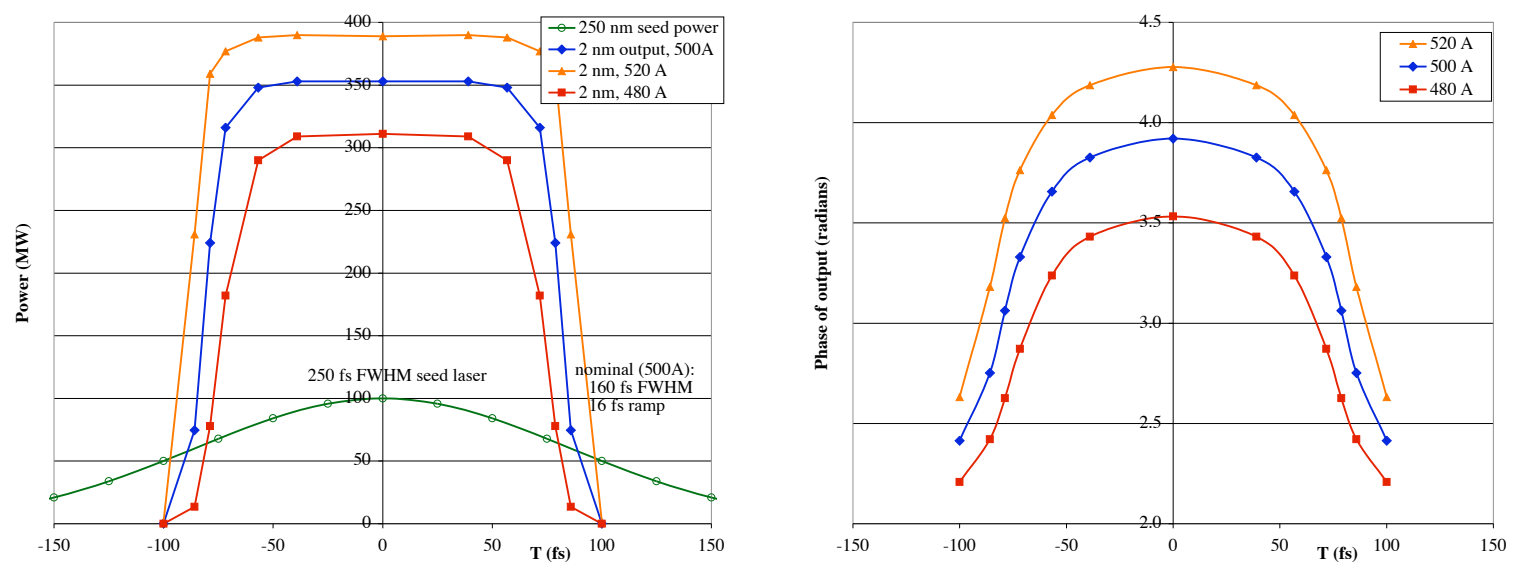

Figure 4. Temporal structure of output power and phase at $3.1 \mathrm{GeV}$, yielding $2.0 \mathrm{~nm}$ radiation from a $250 \mathrm{~nm}$ laser seed using harmonic numbers $4,16,64$, and 128 . Simulations assume an initial laser seed with $100 \mathrm{MW}$ peak power and with FWHM of $200 \mathrm{fs}$, for electron bunch current of $500 \mathrm{~A}$, and $500 \pm 20 \mathrm{~A}$.

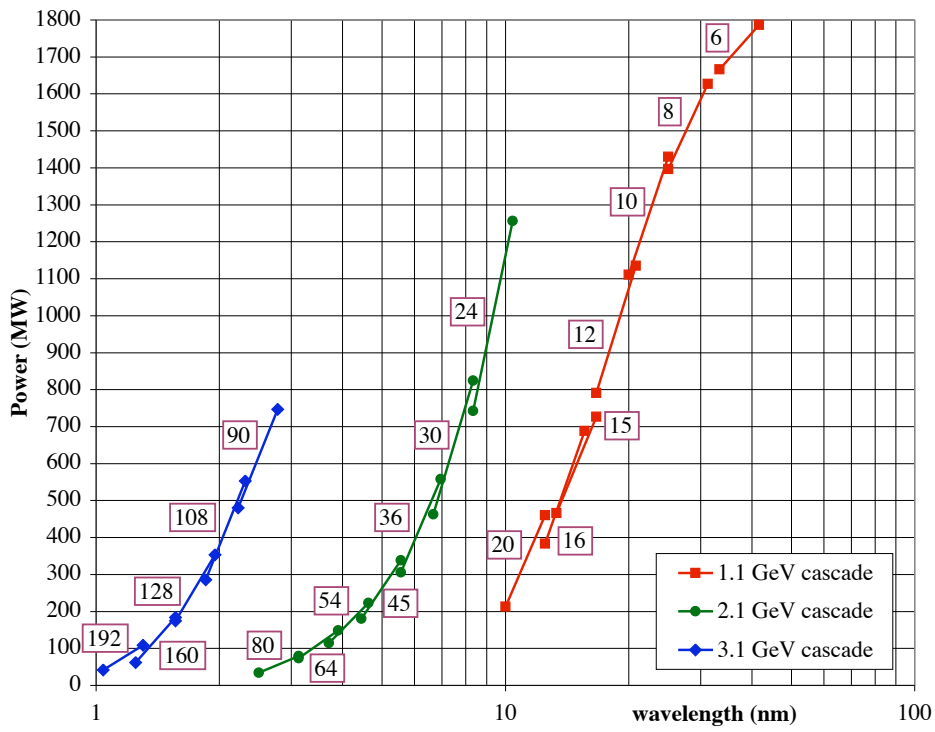

FiguRE 5. Output power as a function of wavelength for the three energy levels, tuned by varying both the input laser frequency and the harmonic number sequence. Total harmonic number is shown in boxes.

to maintain resonance, the output radiation will vary from $3.1 \mathrm{~nm}-3.9 \mathrm{~nm}$ wavelength. To go to longer wavelengths, the harmonic sequence can then be altered to $6-18-54$ and the undulators retuned to match a $200 \mathrm{~nm}$ laser seed, yielding a $3.7 \mathrm{~nm}$ output wavelength. Again, the wavelength of the seed laser can be swept up to $250 \mathrm{~nm}$, and the undulators adjusted to yield output radiation in the range $3.7 \mathrm{~nm}-4.6 \mathrm{~nm}$. By overlapping the range of wavelengths produced using many different harmonic sequences, a continuous range of wavelengths becomes available. Sweeping the seed laser frequency will be simpler than changing the sequence of harmonic numbers, because the changes in undulator parameters required to adjust for small, continuous shifts in radiation wavelength can be approximated by a linear relationship. 


\section{Output Laser Quality}

The transverse structure of the output radiation is predominantly in the fundamental Gaussian mode, with some components at higher harmonics. The transverse profile can be characterized by the $M^{2}$ parameter, where $M^{2} \geq 1$ and $M^{2}=1$ only for a pure Gaussian mode. Values of $2-3$ are generally considered to be reasonable quality for lasers. The transverse quality of the output radiation only degrades significantly when the final undulator is operating at or beyond the emittance limit, so that for the $3.1 \mathrm{GeV}, 1 \mathrm{~nm}$ case, $M^{2} \simeq 1.76$, and for the $2.1 \mathrm{GeV}, 2.5 \mathrm{~nm}$ case, $M^{2} \simeq 1.27$.

In all cases, the temporal profile of the output radiation is a flat-top pulse with a sharp drop in output power once the seed laser falls below a threshold value. The flat-top profile results in a minimum factor of 2 reduction in peak brilliance compared with a Gaussian profile. The phase variation is roughly quadratic in time, corresponding to a positive chirp in frequency. The total phase variation is typically 1 or 2 radians; this is enough to correspond to a modest $10-50 \%$ increase in bandwidth compared to the minimal bandwidth for the given power profile, with a corresponding decrease in peak brilliance. Background levels due to shot noise from the beam vary depending on the output wavelength, but are typically of the order of $40 \mathrm{~kW}$. For a 2 ps electron bunch, that corresponds to a total energy per bunch of $0.08 \mu \mathrm{J}$ due to shot noise, which is significantly below the energy per pulse produced coherently.

For a perfect input seed laser, the variations in phase with time yield only a slight degradation in FEL performance in terms of the peak brilliance. Because the output radiation depends on the structure of the laser seed over only a small time interval, the influence of long time scale jitter in the input seed laser can be inferred from the previous calculations. Further questions about the influence of fluctuations in laser phase at short and medium time scales require full time dependent simulations, and will be looked at in the future. W.M. Fawley has already looked into this issue using the GINGER FEL code [3]. Fluctuations in the peak laser power are more straightforward to calculate; a $10 \%$ variation in the peak laser power, with the same FWHM in time, yields variations of up to $17 \%$ in output power for the $2.1 \mathrm{GeV}, 2 \mathrm{~nm}$ cascade, which is the most sensitive case. For the $1.1 \mathrm{GeV}$ cascade, on the other hand, the resulting variations are less than $10 \%$.

\section{Beam Quality Requirements}

TABLE 5. Dependence of peak power on beam quality. The output from the nominal design is compared with configurations tuned for $400 \mathrm{~A}$ beam current, $3 \mu \mathrm{m}$ emittance, or $\sigma_{\gamma}=0.59$.

\begin{tabular}{|l|cccc|}
\hline Peak power in MW: & & & & \\
& $\begin{array}{c}1.1 \mathrm{GeV} \\
10 \mathrm{~nm}\end{array}$ & $2.1 \mathrm{GeV}$ & $3.1 \mathrm{GeV}$ & $3.1 \mathrm{GeV}$ \\
& & & & \\
Beam Parameters: & & & & \\
\hline Nominal & 213 & 34.6 & 353 & 41.4 \\
$I=400 \mathrm{~A}$ & 104 & 13.6 & 138 & 9.3 \\
$\epsilon_{N}=3.0 \mu \mathrm{m}$ & 84 & 9.8 & 103 & 7.2 \\
$\sigma_{\gamma}=0.59$ & 78 & 2.4 & 11 & 4.8 \\
\hline
\end{tabular}

The performance of the FELs is constrained by the energy spread, emittance, and peak current which can be delivered to the undulators. A degradation in any of these parameters will reduce the production of high-energy photons. In Table 5, the results from the nominal beam parameters are compared with harmonic cascades that have been reoptimized for various changes in the electron beam; either the current has been reduced by $20 \%$ to $400 \mathrm{~A}$, or the emittance has been increased by $50 \%$ to $3 \mu \mathrm{m}$, or the energy spread has been increased by $50 \%$ to $\sigma_{\gamma} / \gamma=0.59$. The highest photon energy for each cascade is shown, as well as the $2.0 \mathrm{~nm}, 3.1 \mathrm{GeV}$ case. Typically, when the peak power is lowered, the threshold on the initial laser power is raised, resulting in shorter pulses. Therefore, the reduction in the total number of photons is even greater. The lengths of the undulators were left unchanged in each case; to accomodate beam parameters below the nominal values, it may be desirable to lengthen some of the undulators. The energy spread is a particular worry because the bunching parameter within each harmonic generation section typically falls off exponentially with the ratio $\left(\sigma_{\gamma} / \gamma_{M}\right)^{2}$, where $\gamma_{M}$ is the energy modulation generated in the modulating 
undulator. When the ratio is small, this effect is not noticeable, but it can rapidly degrade the performance once the energy spread increases beyond a certain threshold.

\section{Sensitivity Studies}

TABle 6. Sensitivity studies: dependence of peak power on electron beam current, emittance, and energy spread, for different harmonic cascades.

\begin{tabular}{|c|c|c|c|c|}
\hline Peak power in MW: & $\begin{array}{l}1.1 \mathrm{GeV} \\
10 \mathrm{~nm}\end{array}$ & $\begin{array}{l}2.1 \mathrm{GeV} \\
2.5 \mathrm{~nm}\end{array}$ & $\begin{array}{l}3.1 \mathrm{GeV} \\
2.0 \mathrm{~nm}\end{array}$ & $\begin{array}{l}3.1 \mathrm{GeV} \\
1.0 \mathrm{~nm}\end{array}$ \\
\hline Nominal & 213 & 34.6 & 353 & 41.4 \\
\hline$I=480 \mathrm{~A}$ & 185 & 29.3 & 311 & 38.1 \\
\hline$I=520 \mathrm{~A}$ & 237 & 38.6 & 389 & 45.8 \\
\hline$\epsilon_{N}=2.2 \mu \mathrm{m}$ & 172 & 25.5 & 278 & 33.0 \\
\hline$\epsilon_{N}=1.8 \mu \mathrm{m}$ & 260 & 39.5 & 428 & 51.3 \\
\hline$\sigma_{\gamma}=0.43$ & 189 & 29.1 & 323 & 34.8 \\
\hline$\sigma_{\gamma}=0.35$ & 234 & 38.9 & 342 & 48.7 \\
\hline
\end{tabular}

The sensitivity studies described here focus on the dependence of the output radiation on variations in electron beam properties: in particular, on emittance, energy spread, and current. Additionally, the effect of misalignments, both in position and angle, are considered. Variation in the peak power is used as a measure of the overall sensitivity of the harmonic cascade to beam parameters. In all of the following examples, the harmonic cascade is tuned for the nominal case, and no settings are altered as the input beam properties vary. The results of shifting the beam current by $\pm 4 \%$ have already been shown in the above figures, including the variation in temporal profile. In Table 6 , the results of the sensitivity studies are summarized. The highest photon energy for each cascade is shown, as well as the $3.1 \mathrm{GeV}, 2.0 \mathrm{~nm}$ case. The variation in electron beam properties is here taken to be uniform across the bunch, which should exaggerate the overall effect of fluctuations because there will tend to be a partial cancellation of errors in each stage of the cascade. There is no readjustment of the lattice. Typically, when the peak power is lowered, the threshold on the initial laser power is raised, resulting in shorter pulses. The dependence of the pulse duration on electron beam properties is very similar to the dependence of peak power, and so the sensitivity is effectively doubled for applications which depend on total energy per pulse. The output power is most sensitive to the beam current; because the cascade is designed to be short, the extra power produced in each radiator in the case where the current is above the nominal value of $500 \mathrm{~A}$ contributes directly to the final output power. Longer beamlines, by contrast, can be tuned to run optimally at a particular beam current, at the expense of reducing the actual output power. The beam emittance also has a strong effect even when the FELs are not emittance-limited, because the laser and electron beams tend to have the same size in later stages; increasing the beam emittance also increases the beam size, which reduces the overall coupling. The harmonic cascade is slightly less sensitive to energy spread, and for the $2.0 \mathrm{~nm}$ example the cascade performance is actually close to being maximized by the nominal energy spread.

While phase deviations within the laser seed are a potential problem, fluctuations in laser power should not have a strong effect on the output seed. In particular, the output power is a fairly flat function of the input power, except for a small interval where the laser power is at a threshold for the harmonic cascade to work. As long as the location of this threshold crossing is kept stable, the total energy per pulse produced by the final radiator should be fairly insensitive to variations in power of the laser seed.

Finally, we consider the effect of misalignments. In Table 7, the effect of undulator displacements and misalignments in angle is shown for various harmonic cascades. Each simulation incorporates a set of misalignments for each undulator; the misalignments can be in either $x$ or $y$, and either positive or negative, and the magnitude of the misalignments are as shown. The dominant effect of misalignments is to reduce the overlap between the electron bunch and laser beams, especially for the low-wavelength sections where the electron bunch radius and laser beam spot size are comparable. The example with $1 \mathrm{~nm}$ output radiation is 
TABLE 7. Output power under conditions of misalignments in undulator position and angle, for harmonic cascades producing $1,2,2.5$, and $10 \mathrm{~nm}$ radiation from the final undulator.

\begin{tabular}{|l|cccc|}
\hline Peak power in MW: & \multicolumn{4}{|c}{} \\
& $\begin{array}{c}1.1 \mathrm{GeV} \\
10 \mathrm{~nm}\end{array}$ & $\begin{array}{c}2.1 \mathrm{GeV} \\
2.5 \mathrm{~nm}\end{array}$ & $\begin{array}{c}3.1 \mathrm{GeV} \\
2.0 \mathrm{~nm}\end{array}$ & $\begin{array}{c}3.1 \mathrm{GeV} \\
1.0 \mathrm{~nm}\end{array}$ \\
Misalignments: & & & & \\
\hline None & 213 & 34.6 & 353 & 41.4 \\
\hline Position: & & & & \\
$10 \mu \mathrm{m}$ & 210 & 32.8 & 330 & 35.2 \\
$20 \mu \mathrm{m}$ & 198 & 28.7 & 269 & 23.8 \\
$30 \mu \mathrm{m}$ & 179 & 23.1 & 176 & 10.7 \\
$40 \mu \mathrm{m}$ & 155 & 16.9 & 74.0 & 2.31 \\
\hline Angle: & & & & \\
$2 \mu \mathrm{rad}$ & 214 & 33.9 & 324 & 37.8 \\
$4 \mu \mathrm{rad}$ & 212 & 31.9 & 253 & 26.8 \\
$6 \mu \mathrm{rad}$ & 208 & 28.7 & 161 & 12.4 \\
$8 \mu \mathrm{rad}$ & 200 & 24.2 & 78.7 & 3.8 \\
$10 \mu \mathrm{rad}$ & 190 & 18.7 & 26.5 & 0.93 \\
\hline
\end{tabular}

the most sensitive. For this cascade, there is a reduction of almost $50 \%$ in peak power when the undulators are displaced by $20 \mu \mathrm{m}$. The other examples show a similar behavior, with performance degrading significantly at or above $20 \mu \mathrm{m}$ displacements. Misalignments in angle also reduce the peak power; the $1.0 \mathrm{~nm}$ example is the most sensitive, exhibiting a drop of $50 \%$ in peak power for misalignments of $4 \mu \mathrm{rad}$. The $10 \mathrm{~nm}$ cascade is the least sensitive, and does not suffer a $50 \%$ reduction in peak power until the undulator displacements reach $60 \mu \mathrm{m}$ or the angles reach $20 \mu \mathrm{rad}$. For comparison, the RMS radius of the electron bunch is $80-110$ $\mu \mathrm{m}$, and the RMS angle (subtracting out the undulator motion) is $4-8 \mu \mathrm{rad}$. For misalignments in both position and angle, the power drop grows quadratically with the size of the misalignments.

The fluctuations in the output laser phase caused by errors in the angle of the undulators are more pronounced than that caused by errors in positioning. Thus, for the $1 \mathrm{~nm}$ cascade, deviations of order $20 \mu \mathrm{m}$ reduce the output power by almost a factor of 2 , and change the phase by less than a radian, while deviations of order $4 \mu \mathrm{rad}$ also reduce the power by a factor of 2 , but change the phase by 2 radians. Even small angles of this scale can change the path length by of order a nanometer, causing significant phase changes.

Electron beam misalignments have a different scaling, because their effect is predominantly felt at the first modulator due to mismatch between the electron beam and laser seed. This effect is comparable to misalignments in the seed laser itself, because in later stages the orbit deviations in the electron beam evolve slowly enough that the output from every radiator will still overlap the electron beam in the following modulator. For example, in the $3.1 \mathrm{GeV}, 1.0 \mathrm{~nm}$ cascade, the peak output power is nearly flat until the deviation is greater than $100 \mu \mathrm{m}$, and then abruptly falls off. This mimicks the effect of reducing the seed laser power, because the reduced overlap between the electron and laser beams produces a smaller energy modulation in the first undulator. On the other hand, errors in the initial beam angle have a more gradual effect similar to errors in undulator angles, with a deviation of $7 \mu \mathrm{rad}$ causing a $50 \%$ reduction in power. This sensitivity results from the fact that the length of the harmonic cascade is roughly half of a betatron period. If the electron beam starts off with a misalignment of $7 \mu \mathrm{rad}$, by the time the beam enters the final radiator it is near its maximum displacement at $140 \mu \mathrm{m}$ off-axis. The displacement in the final undulator alters the resonance condition, which is particularly sensitive to displacements because of the short periodicity and long total length of this undulator. Orbit errors caused by the delay line are also similar to errors in undulator alignment. 


\section{AcKnowledgements}

I wish to thank A. Zholents and J. N. Corlett for their guidance and support in pursuing this research, and W. M. Fawley for many fruitful discussions in which he offered his insights into computer simulations and general FEL physics.

This work was supported by the Director, Office of Science, of the U.S. Department of Energy under Contract No. DE-AC03-76SF00098.

\section{REFERENCES}

[1] W. A. Barry, W. A. Barletta, J. M. Byrd, J. N. Corlett, et al., Feasibility study for a recirculating linac-based facility for femtosecond dynamics, Report LBNL-51766, LBNL, December 2002.

[2] J. N. Corlett, W. A. Barletta, S. DeSantis, L. Doolittle, et al., A recirculating linac-based facility for ultrafast X-ray science, Proceedings of the Paritcle Accelerator Conference, 2003 (PAC2003), IEEE, vol. 1, 2003, Piscataway, NJ, pp. $186-188$.

[3] W. M. Fawley, W. A. Barletta, J. N. Corlett, and A. Zholents, Simulations studies of an XUV/soft X-ray harmonic-cascade FEL for the proposed LBNL recirculating linac, Proceedings of the Paritcle Accelerator Conference, 2003 (PAC2003), IEEE, vol. 2, 2003, Piscataway, NJ, pp. 923-925.

[4] L.-H. Yu, M. Babzien, I. Ben-Zvi, L. F. DiMauro, et al., High-gain harmonic-generation free-electron laser, Science 289 (2000), 932-934.

[5] L.-H. Yu, L. F. DiMauro, A. Doyuran, W. S. Graves, et al., First ultraviolet high gain harmonic generation free electron laser, Physical Review Letters 91 (2003), 074801.

[6] R. Bonifacio, C. Pellegrini, and L. M. Narducci, Collective instabilities and high-gain regime in a free electron laser, Optics Communications 50 (1984), 373-378.

[7] K. Halbach, Permanent magnet undulators, J. de Physique: Colloque 44 (1983), no. C1, 211-216.

[8] P. Elleaume, J. Chavanne, and B. Faatz, Design considerations for a 1 A SASE undulator, Nucl. Instr. Methods A 455 (2000), 503-523.

[9] S. Reiche, GENESIS 1.3: a fully 3D time-dependent FEL simulation code, Nucl. Instr. Methods A 429 (1999), $243-248$.

[10] W. M. Fawley, A user manual for GINGER and its postprocessor XPLOTIN, Report LBNL-49625, LBNL, February 2002. 